\title{
Resolution of inflammation in obesity-induced liver disease
}

\section{Bibiana Rius ${ }^{1}$, Cristina López-Vicario ${ }^{1}$, Ana González-Périz ${ }^{1,2}$, Eva Morán-Salvador ${ }^{1}$, Verónica García-Alonso ${ }^{1}$, Joan Clària ${ }^{1,2,3}$ * and Esther Titos ${ }^{1,2}$}

\author{
1 Department of Biochemistry and Molecular Genetics, Hospital Clínic, Centre Esther Koplowitz, Institut d'investigacions Biomèdiques \\ August Pi i Sunyer, Barcelona, Spain \\ ${ }^{2}$ CIBERehd, Barcelona, Spain \\ ${ }^{3}$ Department of Physiological Sciences I, University of Barcelona, Barcelona, Spain
}

\section{Edited by:}

Janos G. Filep, University of Montreal, Canada

\section{Reviewed by:}

Giamila Fantuzzi, University of Illinois at Chicago, USA

Hiroki Yoshida, Saga University

Faculty of Medicine, Japan

*Correspondence:

Joan Clària, Department of Biochemistry and Molecular Genetics, Hospital Clínic, Centre Esther Koplowitz, Institut d'investigacions Biomèdiques August Pi i Sunyer, Villarroel 170, Barcelona 08036, Spain. e-mail: jclaria@clinic.ub.es
Low-grade inflammation in adipose tissue is recognized as a critical event in the development of obesity-related co-morbidities. This chronic inflammation is powerfully augmented through the infiltration of macrophages, which together with adipocytes, perpetuate a vicious cycle of inflammatory cell recruitment and secretion of free fatty acids and deleterious adipokines that predispose to greater incidence of metabolic complications. In the last decade, many factors have been identified to contribute to mounting unresolved inflammation in obese adipose tissue. Among them, pro-inflammatory lipid mediators (i.e., leukotrienes) derived from the omega-6 polyunsaturated arachidonic acid have been shown to play a prominent role. Of note, the same lipid mediators that initially trigger the inflammatory response also signal its termination by stimulating the formation of anti-inflammatory signals. Resolvins and protectins derived from the omega-3 polyunsaturated docosahexaenoic and eicosapentaenoic acids have emerged as a representative family of this novel class of autacoids with dual anti-inflammatory and pro-resolving properties that act as "stop-signals" of the inflammatory response. This review discusses the participation of these endogenous autacoids in the resolution of adipose tissue inflammation, with a special emphasis in the amelioration of obesity-related metabolic dysfunctions, namely insulin resistance and non-alcoholic fatty liver disease.

Keywords: obesity, omega- 6 fatty acids, eicosanoids, omega-3 fatty acids, resolvins, stromal-vascular macrophages, Kupffer cells

\section{RESOLUTION OF INFLAMMATION: CIRCUITS AND CHEMICAL MEDIATORS}

Inflammation plays a vital role in host defense against invasive pathogens and tissue and wound repair. Inflammation is part of the innate immune response and is initiated by a cascade of signals in response to an infection or injury that leads to the recruitment of specialized inflammatory cells, particularly neutrophils (PMN), into injured tissue to neutralize and eliminate the injurious stimuli (Barton, 2008; Chen and Nuñez, 2010). The innate immune response not only acts as the first line of defense against an insult, but it also provides the necessary signals to instruct the adaptive immune system for an effective response to deal with the noxious agent. Although inflammation is important in eradication of pathogens, unresolved, chronic inflammation that occurs when the offending agent is not removed or contained is detrimental to the host, resulting in tissue damage, fibrosis, and loss of function (Barton, 2008; Chen and Nuñez, 2010).

Since unresolved inflammation is detrimental to the host, higher organisms have evolved protective mechanisms to ensure resolution of the inflammatory response in a specific time-limited manner (Serhan etal., 2008). Once considered a mere passive process of dilution, resolution is today envisioned as a highly orchestrated process coordinated by a complex regulatory network of cells and mediators. This novel insight offers the possibility to harness resolution factors that clear inflammation and use them to ameliorate the pathologies associated with chronic inflammation.
This has the benefit to avoid any unwanted side-effect observed during the long-term therapy with anti-inflammatory drugs such as cyclooxygenase (COX) inhibitors. COX inhibitors, like aspirin (ASA) or ibuprofen, can cause gastrointestinal irritation and renal damage when used in high doses (Wallace and Vong, 2008). Although at first glance selective COX-2 inhibitors looked like to overcome NSAID toxicity on the gastrointestinal tract, COX2 inhibitors as Vioxx were later withdrawn from the market for their increased risk of cardiovascular thrombotic events (Wallace and Vong, 2008). For this reason, the search for novel targets and the identification of molecular circuits and chemical mediators involved in resolution represent a priority in anti-inflammatory therapy.

Among the molecules that facilitate resolution, lipid mediators derived from the metabolism of essential polyunsaturated fatty acids have attracted most attention. The first recognized family of specialized pro-resolving mediators (SPM) was the lipoxins (LXs). LXs are conjugated trihydroxytetraene-containing eicosanoids generated from endogenous sources of the omega- 6 arachidonic acid (Serhan, 2002). A major route of transcellular LX biosynthesis is initiated by 15-lipoxygenase (15-LO) forming $15 S$-hydroxyeicosatetraenoic acid (15S-HETE), which is rapidly converted to $\mathrm{LXA}_{4}$ by 5 -LO (Figure 1; Serhan et al., 1984). Another major route of transcellular LX biosynthesis is the generation of 15-epi-LXs through a circuit initiated by acetylation of COX-2 by ASA (Clària and Serhan, 1995). In this route, when ASA inhibits 


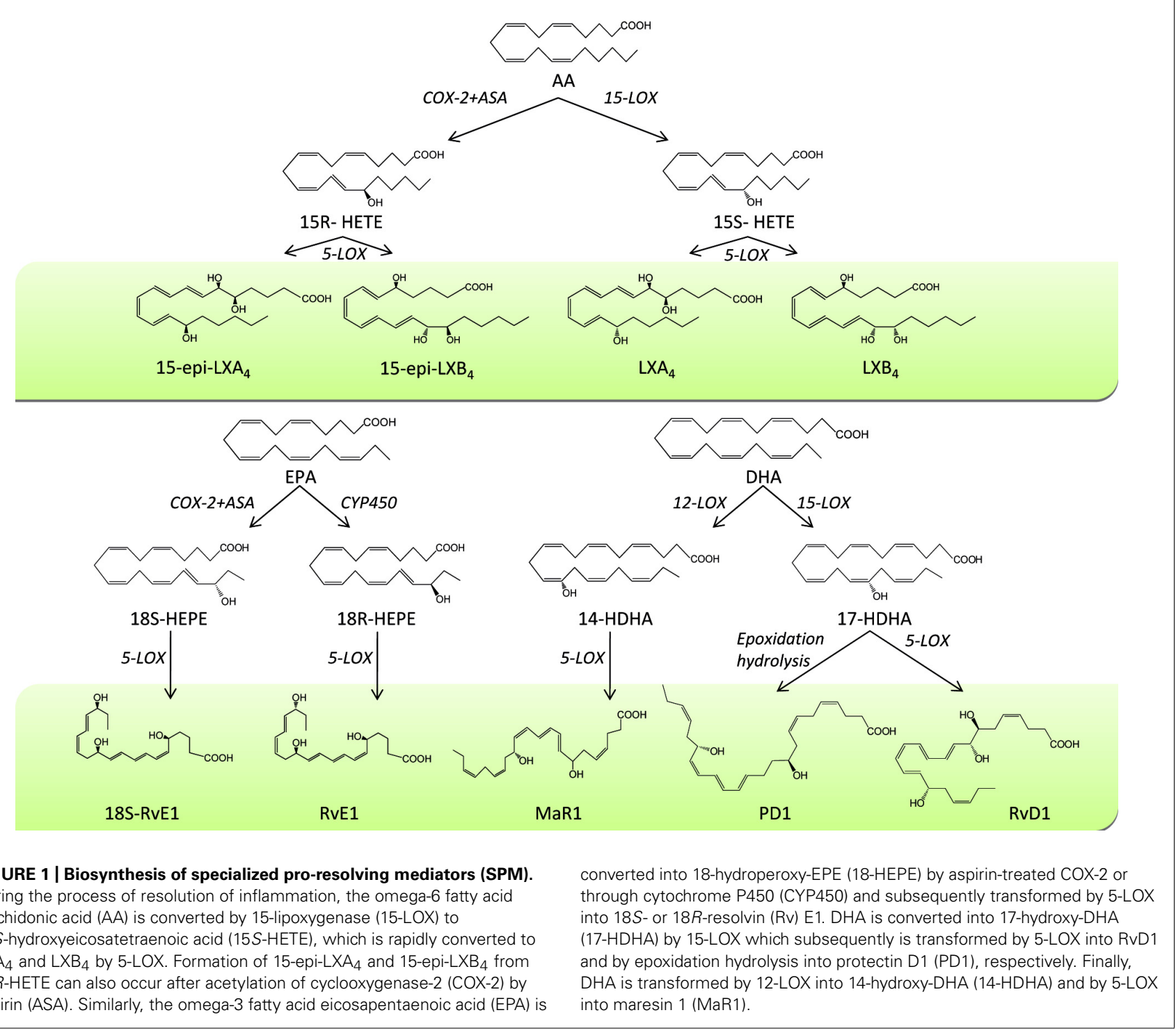

prostaglandin (PG) formation in cells bearing a cytokine-induced COX-2, the resulting ASA-acetylated COX-2 converts arachidonic acid into 15R-HETE. Subsequently, 15R-HETE is transformed by 5 -LO of activated neutrophils into 15-epi-LXs, which carry the carbon-15 alcohol in the $R$ configuration, instead of the $S$ as in the native LXs (Figure 1; Clària and Serhan, 1995). These SPM act as "stop-signals" for inflammation and inhibit leukocyte chemotaxis, adhesion to and transmigration across endothelial monolayers in response to $\mathrm{LTB}_{4}$ (Serhan et al., 2008). LX stable analogs inhibit in vivo $\mathrm{LTB}_{4}$-induced leukocyte rolling, adherence, margination and extravasation and when applied topically to mouse ears they dramatically inhibit leukocyte infiltration and vascular permeability (Serhan et al., 2008).

Resolvins are the second family of SPM with recognized anti-inflammatory and pro-resolving properties. Resolvins are endogenous lipid mediators generated from the omega-3 docosahexaenoic acid (DHA) and eicosapentaenoic acid (EPA). They were initially identified using a lipidomics-based approach that combined liquid chromatography and tandem mass spectrometry within self-limited inflammatory exudates captured during the "spontaneous resolution" phase of acute inflammation (Serhan et al., 2000, 2002). Resolvins are classified into D- and E-series in accordance with their biosynthetic precursor, either DHA or EPA, respectively. Schematically, resolvin biosynthesis is initiated by 15 -LO which transforms endogenous sources of DHA into $17 S$-hydroxy-DHA which is further transformed by leukocyte 5-LO into resolvin (Rv) D1 and RvD2 (Figure 1; Hong et al., 2003). Endothelial cells expressing COX-2 treated with aspirin also transform DHA into 17R-hydroxy-DHA which is further converted by $5-\mathrm{LO}$ into $17 R-\mathrm{RvD} 1$ (Figure 1; Serhan etal., 2000, 2002). DHA can also be metabolized into a dihydroxy-containing derivative via an intermediate epoxide that opens via hydrolysis and subsequent rearrangements to form protectin (PD) 1 (Figure 1; Serhan etal., 2000, 2002; Hong et al., 2003). Similarly, RvE1 biosynthesis is initiated when EPA is converted to 18-hydroperoxy-EPE by aspirin-treated COX-2 
or through cytochrome P450 activity (Serhan etal., 2000; Haas-Stapleton etal., 2007). By transcellular biosynthesis, 18hydroperoxy-EPE is transformed by 5 -LO of neighboring leukocytes into RvE1 via a 5(6)epoxide intermediate (Figure 1; Serhan et al., 2000, 2002).

RvD1, RvD2, PD1, and RvE1 are potent SPM, which contrary to their metabolic substrates, DHA and EPA, exert their biological actions at the nanomolar range. Indeed, the potency of these SPM is notable with concentrations as low as $10 \mathrm{nM}$ producing a $50 \%$ reduction in PMN transmigration. Two receptors (ALX/FPR2 and GPR32) have been shown to transmit RvD1 signals (Krishnamoorthy et al., 2010), whereas a G-protein coupled receptor (ChemR23) signals for RvE1 (Arita et al., 2005a). The full structural elucidation, stereochemical assignment and biological actions for these compounds were first completed in RvE1. RvE1 was readily shown to decrease PMN infiltration and T cell migration, reduce tumor necrosis factor (TNF) $\alpha$ and IFN $\gamma$ secretion, inhibit chemokine formation, and block interleukin (IL)-1-induced NF- $\mathrm{B}$ activation (Schwab et al., 2007; Bannenberg and Serhan, 2010). RvE1 was also shown to stimulate macrophage phagocytosis of apoptotic PMN and to be a potent counter-regulator of L-selectin expression (Schwab et al., 2007; Dona et al., 2008). RvE1 displayed potent anti-inflammatory actions in vivo, protecting mice against experimental periodontitis, colitis, peritonitis, and brain ischemia-reperfusion (Arita et al., 2005b; Bannenberg and Serhan, 2010). A RvE1-initiated resolution program for allergic airway response was identified by Haworth et al. (2008). Similarly, RvD1 and RvD2 were reported to reduce inflammatory pain, block IL-1 $\beta$ transcripts induced by TNF $\alpha$ in microglial cells and function as potent regulators limiting PMN infiltration into inflamed brain, skin, and peritoneum (Hong et al., 2003; Sun et al., 2007). $\mathrm{RvD} 2$ in particular has been shown to be a potent endogenous regulator of excessive inflammatory responses in mice with microbial sepsis (Spite et al., 2009). Moreover, PD1 has been reported to exert protective actions in acute models of inflammation by blocking PMN migration and infiltration into the inflammatory site (Serhan etal., 2006). Finally, these SPM expedite the resolution process by paving the way for monocyte migration and their differentiation to phagocytosing macrophages, which remove dead cells (efferocytosis) and then terminate the inflammatory response by promoting macrophage efflux into lymphatics (Schif-Zuck et al., 2011).

\section{RESOLUTION OF ADIPOSE TISSUE INFLAMMATION IN OBESITY}

Abdominal obesity and insulin resistance are the predominant underlying risk factors for the metabolic syndrome and related co-morbidities such as type 2 diabetes, dyslipidemia, and nonalcoholic fatty liver disease (Elks and Francis, 2010). A wealth of evidence indicates that metabolic disorders associated with obesity are initiated by the presence of a chronic "low-grade" state of inflammation in the adipose tissue (Ferrante, 2007; Elks and Francis, 2010). This "low-grade" inflammatory state is aggravated by the recruitment of inflammatory cells, mainly macrophages in the adipose tissue (Ferrante, 2007; Elks and Francis, 2010). As a consequence of this unresolved inflammatory response, the production of pro-inflammatory adipokines [i.e., IL-6, TNF $\alpha$, and monocyte chemotactic protein-1 (MCP-1)] is increased while the secretion of adiponectin, an anti-inflammatory and insulinsensitizing adipokine, is reduced (Figure 2A; Ferrante, 2007; Elks and Francis, 2010). In addition to adipokines, the formation of pro- and anti-inflammatory lipid mediators is also severely deregulated in obesity. Indeed, we have recently demonstrated that the production of SPM (i.e., RvD1 and PD1 and the metabolic precursors 14-HDHA, 17-HDHA, 18-HEPE) is deficient in inflamed obese adipose tissue (Clària et al., 2012). Whether the response to these mediators is also impaired and whether this SPM deficit is a generalized property of obese tissues are open questions that need to be addressed.

Adipose tissue inflammation is also driven by the activation of classical pro-inflammatory pathways such as arachidonate 5LO. Indeed, over-expression of FLAP is a common finding in adipose tissue of patients and animals with obesity and insulin resistance (Kaaman et al., 2006; Horrillo et al., 2010). Moreover, linkage studies have identified 5-LO as a gene with pleiotropic actions on adipose fat accumulation and pancreatic function (Mehrabian et al., 2008). The ability of adipose tissue to generate 5-LO-derived products has recently been challenged by Horrillo et al. (2010). These authors have demonstrated the presence of all enzymes necessary for the formation of 5-LO products (5LO, FLAP, $\mathrm{LTA}_{4}$ hydrolase, and $\mathrm{LTC}_{4}$ synthase) as well as all receptors involved in leukotriene (LT) signaling (BLT1, BLT2, CysLT1, and CysLT2) in adipose tissue of both lean and obese mice (Horrillo et al., 2010). Importantly, adipose tissue samples from obese mice showed increased formation of 5-LO products, mainly $\mathrm{LTB}_{4}$ (Horrillo et al., 2010). Similar findings have been reported in visceral adipose tissue from obese Zucker rats (Chakrabarti et al., 2011). An important observation of the study by Horrillo et al. (2010) was that $\mathrm{LTB}_{4}$ unequivocally triggered an inflammatory response in adipose tissue by inducing the nuclear translocation of $\mathrm{p} 50$ and $\mathrm{p} 65$ subunits of NF- $\kappa$ B. Secondary to $\mathrm{LTB}_{4}$-induced NF- $\kappa \mathrm{B}$ activation, there was an enhanced release of MCP-1 and IL-6, which directly connect adipose tissue inflammation with insulin resistance and hepatic steatosis (Horrillo et al., 2010). The physiological consequences of these changes in adipose tissue function were corroborated in vivo by observing that either pharmacological inhibition of the 5-LO pathway or genetic deletion of Alox5, the gene coding for 5-LO, alleviate insulin resistance and hepatic steatosis in obese animals (Horrillo et al., 2010; Martínez-Clemente et al., 2010).

In sharp contrast to the pro-inflammatory actions for the most part of omega-6-derived products, omega-3-derived lipid mediators act as "braking signals" of the persistent vicious cycle leading to unremitting inflammation in obese adipose tissue. Endres etal. (1989) were the first to demonstrate antiinflammatory properties of the omega- 3 fatty acids. Since then, supplementation of omega-3 fatty acids has proven to exert overall benefits in obesity and metabolic syndrome. In a recent series of experiments, González-Périz et al. (2009) have demonstrated that administration of an omega-3-enriched diet to ob/ob mice, an experimental model of obesity and fatty liver disease, resulted in increased adiponectin levels and reduced insulin resistance and hepatic steatosis. These changes occurred in parallel with augmented formation of omega-3-derived SPM in 

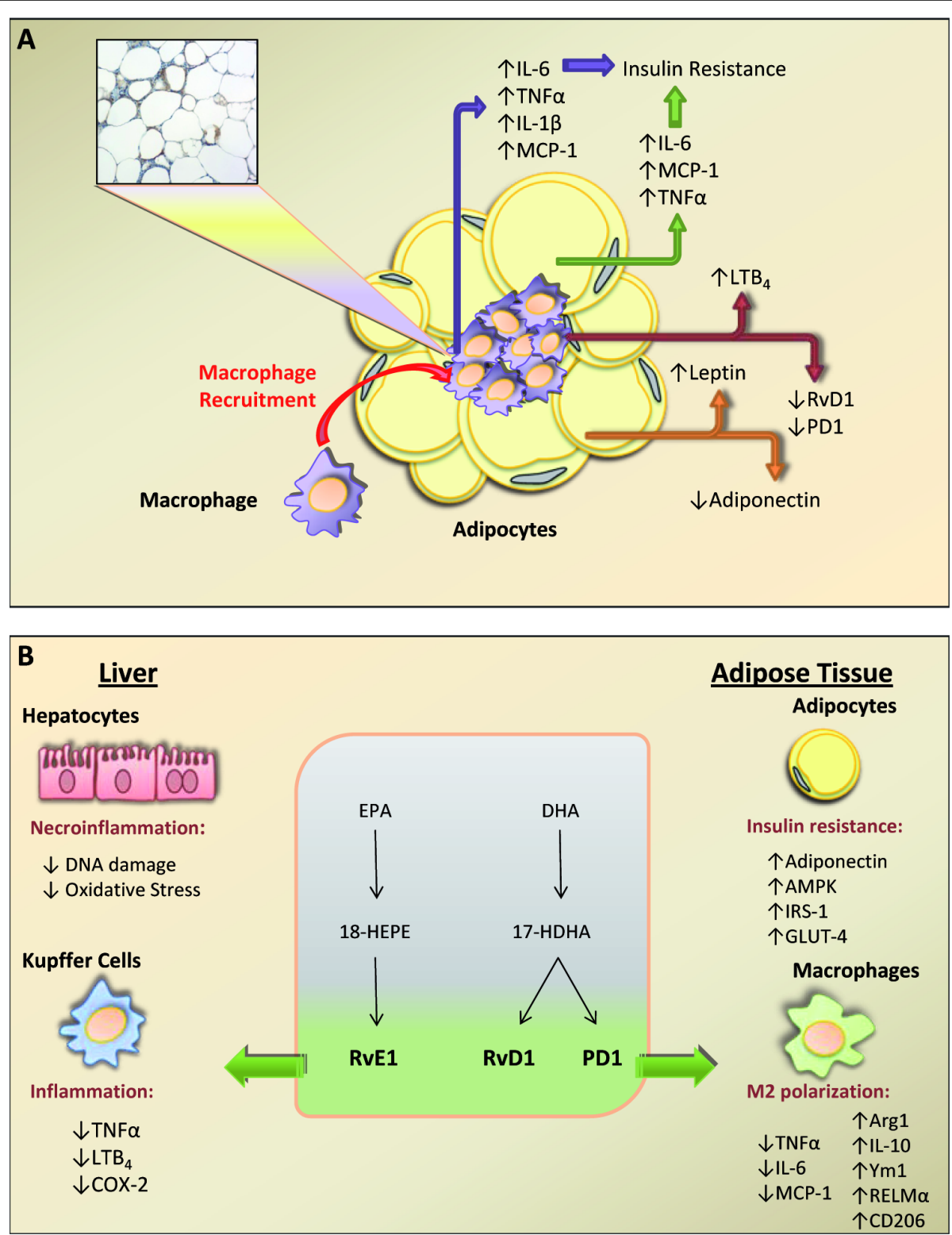

FIGURE 2 | (A) Schematic overview summarizing the cross-talk between macrophages and adipocytes in obese adipose tissue. Obese adipose tissue shows a remarkable infiltration of macrophages which form "crown-like" structures that surround necrotic adipocytes. This recruited macrophages together with hypertrophy and/or hyperplasia of adipocytes produce an aberrant release of pro-inflammatory adipokines [tumor necrosis factor (TNF) $\alpha$, interleukin (IL)-6, IL-1 $\beta$, and monocyte chemotactic protein-1 (MCP-1)] that leads to insulin resistance. Unbalanced formation of pro-inflammatory leukotriene (LT) $B_{4}$ and leptin accompanied by a deficit in anti-inflammatory mediators [i.e., resolvin (Rv) D1, protectin (PD) 1, and adiponectin] contributes to a state of unresolved inflammation in obese adipose tissue. (B) Schematic representation of the protective actions of specialized omega-3-derived mediators on liver and adipose tissue. Eicosapentaenoic acid (EPA) is converted into 18-hydroperoxy-EPE (18-HEPE) and resolvin (Rv) E1, whereas DHA is converted into 17-hydroxy-DHA (17-HDHA) and RvD1 and protectin D1 (PD1). In the liver, these specialized pro-resolving mediators (SPM) protect hepatocytes from DNA damage and oxidative stress and dampen

inflammation by inhibiting TNF $\alpha, \mathrm{LTB}_{4}$, and cyclooxygenase-2 (COX-2) in Kupffer cells. In adipose tissue, SPM exert insulin sensitizing actions by up-regulating adiponectin, AMP-activated protein kinase (AMPK), insulin receptor signaling-1 (IRS-1) and glucose transporter-4 (GLUT-4) in adipocytes and promoting M2 polarization [arginase 1 (Arg1), IL-10, chitinase 3-like 3 (Ym1), resistin-like molecule (RELM)- $\alpha$, and CD206] while inhibiting M1 markers (TNF $\alpha, I L-6$, and MCP-1) in macrophages. adipose tissue, while formation of the omega-6-derived products $\mathrm{PGE}_{2}$, 5-HETE, and $\mathrm{LTB}_{4}$ was significantly inhibited (GonzálezPériz et al., 2009). Along these lines, intraperitoneal injection of nanogram doses of RvE1 elicited significant insulin-sensitizing effects by inducing adiponectin, glucose transporter-4 (GLUT-4) and insulin receptor signaling-1 (IRS-1) expression in adipose tissue and conferred significant protection against hepatic steatosis (González-Périz et al., 2009). Similarly, in leptin receptor-deficient $(d b / d b)$ obese and diabetic mice, nanogram doses of RvD1 improved glucose tolerance, decreased fasting blood glucose, and 
increased insulin-stimulated Akt phosphorylation while reducing the formation of crown-like structures rich in inflammatory macrophages in adipose tissue (Hellmann et al., 2011). Recently, similar beneficial actions have been described for $\mathrm{LXA}_{4}$ in an experimental model of age-associated adipose inflammation (Börgeson et al., 2012).

Omega-3-derived mediators can also induce changes in the status of macrophage polarization toward a pro-resolution phenotype. Tissue macrophages are phenotypically heterogeneous and display an extensive receptor repertoire and a versatile biosynthetic capacity that confer them the plasticity to adapt to different tissue microenvironments (Gordon and Taylor, 2005). Macrophages are broadly characterized by their activation (polarization) state according to the M1/M2 classification system (Mantovani et al., 2007). In this classification, the M1 designation is reserved for classically activated macrophages following stimulation with IFN $\gamma$ and LPS, whereas the M2 designation is applied to the alternatively activated macrophages after in vitro stimulation with IL-4 and IL-13. M1 macrophages secrete high amounts of TNF $\alpha$, IL-1 $\beta$, and IL-6, whereas M2 macrophages dampen pro-inflammatory cytokine levels and promote resolution of inflammation and tissue repair (Gordon and Taylor, 2005). M1/M2 macrophage polarization can be monitored by assessing the expression of selected markers. M1-associated markers include inducible nitric oxide synthase (iNOS) and classical proinflammatory mediators such as TNF $\alpha$, IL-1 $\beta$, IL-6, and MCP-1. In contrast, established M2 markers include scavenger, mannose (CD206) and galactose (Mgl-1) receptors, arginase 1, IL-10, chitinases Ym1 and Ym2, and resistin-like molecule (RELM)- $\alpha$ (Martínez et al., 2009).

In a recent study, Titos et al. (2011) have demonstrated that RvD1 consistently induced M2 polarization in adipose tissue macrophages. These investigators first noticed that DHA did not modify the total number of macrophages in obese adipose tissue, but markedly reduced the percentage of $\mathrm{CD} 11 \mathrm{~b}^{\text {high }} / \mathrm{F} 4 / 80^{\text {high }}$ expressing cells in parallel with the emergence of low-expressing CD11b/F4/80 macrophages, suggesting a phenotypic switch in macrophage polarization. Indeed, these investigators further demonstrated that DHA and RvD1 up-regulated a complete panel of M2 markers including IL-10, CD206, RELM- $\alpha$, and Ym1, and remarkably stimulated arginase 1 expression while promoting non-phlogistic macrophage phagocytosis and attenuating IFN $\gamma /$ LPS-induced Th1 cytokine secretion (Titos etal., 2011). These results were in agreement with those reported by Hellmann etal. (2011), who showed the ability of RvD1 to improve insulin resistance in obese-diabetic mice, by reducing macrophage $\mathrm{F} 4 / 80^{+} \mathrm{CD} 11 \mathrm{c}^{+}$cell accumulation and increasing the percentage of positive F4/80 cells expressing the M2 marker Mgl-1 in adipose tissue. The ability of resolvins to modify macrophage plasticity has also been demonstrated by Schif-Zuck etal. (2011), who reported that administration of RvD1 and RvE1 to peritonitis-affected mice enhanced the appearance of $\mathrm{CD}_{11} \mathrm{~b}^{\text {low }}$ macrophages by reducing the number of engulfmentrelated events required for macrophage deactivation and by reducing the ability of peritoneal macrophages to produce proinflammatory cytokines upon LPS stimulation. As the majority of macrophages that accumulate in obese adipose tissue are M1 inflammatory type, these findings are a strong argument in favor of the pro-resolution actions of omega-3-derived mediators in obese adipose tissue.

\section{RESOLUTION OF OBESITY-INDUCED STEATOHEPATITIS}

Lipids, adipokines, and other soluble factors released by inflamed adipose tissue have a direct impact on other insulin-sensitive tissues, especially on the liver. In fact, both adipose and hepatic tissues have immediate access to a vast network of blood vessels that implicate a direct connection between these two tissues. This connection is exemplified by the observation that the circulating fatty acid pool derived from fat is the primary contributor to hepatic steatosis, the initial stage in non-alcoholic steatohepatitis (Donnelly et al., 2005). In this context, adiponectin represents a paradigmatic example of the direct control of adipokines on liver function. Adiponectin, which is an adipokine with potent anti-inflammatory and insulin-sensitizing properties, is a hepatoprotective adipokine lowering hepatic steatosis and insulin resistance and preventing liver fibrosis (Tilg, 2010). Importantly, adiponectin is able to up-regulate the RvE1 receptor ChemR23 in primary human adipocytes, which expression is seriously compromised in human and rodent fatty liver (Wanninger et al., 2012).

To better appreciate how adipose tissue influences hepatic inflammation and the progression from steatosis to steatohepatitis, it is necessary to fully understand the complex cellular architecture of the liver. The hepatic tissue is arranged in a peculiar fenestrated capillary network known as the hepatic sinusoid (Wisse et al., 1996). The morphological features of the hepatic sinusoid provide a unique environment where each single hepatocyte is in close contact with other hepatocytes as well as with non-parenchymal sinusoidal liver cells, including Kupffer cells, endothelial cells, and hepatic stellate cells (Wisse et al., 1996). In terms of inflammation, Kupffer cells, the liver resident macrophages, play the most relevant role and have been classically considered the major sinusoidal cell type involved in hepatic eicosanoid formation (Decker, 1990). Indeed, Kupffer cells express COX-1, COX-2, and 5-LO and generate relevant amounts of $\mathrm{PGE}_{2}, \mathrm{PGI}_{2}, \mathrm{PGF}_{2 \alpha}, \mathrm{PGD}_{2}$, $\mathrm{LTB}_{4}$, and $\mathrm{LTC}_{4} / \mathrm{LTD}_{4} / \mathrm{LTE}_{4}$ (Decker, 1990; Titos et al., 2000, 2003). These resident hepatic macrophages are also able to generate $\mathrm{LXA}_{4}$ from endogenous sources of arachidonic acid or by transcellular biosynthesis from 15S-HETE released by nearby 15LO-containing hepatocytes (Clària and Planagumà, 2005). Unlike $\mathrm{LTB}_{4}$ and $\mathrm{PGE}_{2}$, Kupffer cell-derived $\mathrm{LXA}_{4}$ down-regulates the cytokine-chemokine axis in adjacent hepatocytes (Planagumà et al., 2002).

Liver tissue is also a rich source of omega-3-derived SPM, such as PD1 and its intermediate precursor 17S-HDHA (GonzálezPériz etal., 2006). These SPM produced an amelioration of necroinflammatory liver injury, an effect that was associated with a decrease in hepatic COX-2 expression and $\mathrm{PGE}_{2}$ formation and reduced genotoxic DNA damage and oxidative stress in isolated hepatocytes (González-Périz et al., 2006). More important, these SPM reduced TNF $\alpha$ release in macrophages, recognized as the predominant effector cells involved in the inflammatory cascade leading to hepatocyte damage. A significant down-regulation of 5-LO protein expression was also noticed in macrophages treated 
with 17S-HDHA and in liver tissue from mice receiving DHA in the diet (González-Périz et al., 2006). This is relevant because the presence of an active 5-LO pathway in the liver is restricted to Kupffer cells and its inhibition is linked to lower necroinflammatory liver injury and fibrosis (Titos et al., 2000, 2003, 2005).

\section{SUMMARY}

Obesity and the associated metabolic disorders are characterized by the presence of a chronic "low-grade" inflammatory response in insulin sensitive tissues, in particular adipose tissue and liver. The mechanisms explaining this observation are unknown but unremitting inflammation is likely to be the consequence of an impaired resolution. Resolution of inflammation (the so-called, "catabasis") is not a passive process that simply occurs when the stimulus disappears, but it is a highly regulated process that requires the coordinated action of pro-resolution SPM. Among

\section{REFERENCES}

Arita, M., Bianchini, F., Aliberti, J., Sher, A., Chiang, N., Hong, S., Yang, R., Petasis, N. A., and Serhan, C. N. (2005a). Stereochemical assignment, antiinflammatory properties, and receptor for the omega-3 lipid mediator resolvin E1. J. Exp. Med. 201, 713-722.

Arita, M., Yoshida, M., Hong, S., Tjonahen, E., Glickman, J. N., Petasis, N. A., Blumberg, R. S., and Serhan, C. N. (2005b). Resolvin E1, an endogenous lipid mediator derived from omega-3 eicosapentaenoic acid, protects against 2,4,6-trinitrobenzene sulfonic acidinduced colitis. Proc. Natl. Acad. Sci. U.S.A. 102, 7671-7676.

Bannenberg, G., and Serhan, C. N. (2010). Specialized pro-resolving lipid mediators in the inflammatory response: an update. Biochim. Biophys. Acta 1801, 1260-1273.

Barton, G. M. (2008). A calculated response: control of inflammation by the innate immune system. J. Clin. Invest. 118, 413-420.

Börgeson, E., McGillicuddy, F. C., Harford, K. A., Corrigan, N., Higgins, D. F., Maderna, P., Roche, H. M., and Godson, C. (2012). Lipoxin A4 attenuates adipose inflammation. FASEB J. doi: 10.1096/fj.12-208249 [Epub ahead of print].

Chakrabarti, S. K., Wen, Y., Dobrian, A. D., Cole, B. K., Ma, Q., Pei, H., Williams, M. D., Bevard, M. H., Vandenhoff, G. E., Keller, S. R., Gu, J., and Nadler, J. L. (2011). Evidence for activation of inflammatory lipoxygenase pathways in visceral adipose tissue of obese Zucker rats. Am. J. Physiol. Endocrinol. Metab. 300, E175-E187.

Chen, G. Y., and Nuñez, G. (2010). Sterile inflammation: sensing and reacting to damage. Nat. Rev. Immunol. $10,826-837$.
Clària, J., Dalli, J., Yacoubian, S., Gao, F., and Serhan, C. N. (2012). Resolvin D1 and resolvin D2 govern local inflammatory tone in obese fat. J. Immunol. doi: 10.4049/jimmunol.1101665 [Epub ahead of print].

Clària, J., and Planagumà, A. (2005). Liver: the formation and actions of aspirin-triggered lipoxins. Pros taglandins Leukot. Essent. Fatty Acids 73, 277-282.

Clària, J., and Serhan, C. N. (1995). Aspirin triggers previously unrecognized bioactive eicosanoids by human endothelial cell-leukocyte interaction. Proc. Natl. Acad. Sci. U.S.A. 92, 9475-9479.

Decker, K. (1990). Biologically active products of stimulated liver macrophages (Kupffer cells). Eur. J. Biochem. 192, 245-261.

Dona, M., Fredman, G., Schwab, J. M., Chiang, N., Arita, M., Goodarzi, A., Cheng, G., von Andrian, U. H., and Serhan, C. N. (2008). Resolvin E1, an EPA-derived mediator in whole blood, selectively counterregulates leukocytes and platelets. Blood 112, 848-855.

Donnelly, K. L., Smith, C. I., Schwarzenberg, S. J., Jessurun, J., Boldt, M. D. and Parks, E. J. (2005). Sources of fatty acids stored in liver and secreted via lipoproteins in patients with nonalcoholic fatty liver disease. J. Clin. Invest. 115, 1343-1351.

Elks, C. M., and Francis, J. (2010). Central adiposity, systemic inflammation, and the metabolic syndrome. Curr. Hypertens. Rep. 12, 99-104.

Endres, S., Ghorbani, R., Kelley, V. E., Georgilis, K., Lonnemann, G., van der Meer, J. W., Cannon, J. G., Rogers, T. S., Klempner, M. S., Weber, P. C., Schaefer, E. J., Wolff, S. M., and Dinarello, M. D. (1989).

these, in recent years we have witnessed an emergence of a number of SPM carrying both anti-inflammatory and pro-resolution properties, namely LXs, resolvins, and protectins. A schematic representation of the actions of these lipid mediators on adipose tissue and liver cells is shown in Figure 2B. In summation, these autacoids enhance inflamed adipose tissue catabasis and provide powerful templates for the design of novel therapies to combat the progression of metabolic complications associated with obesity.

\section{ACKNOWLEDGMENTS}

Our laboratory is supported by grants from the Ministerio de Economía y Competitividad (SAF 09/08767 and SAF 12/32789) and is a Consolidated Research Group recognized by the Generalitat de Catalunya (2009SGR1484). CIBERehd is funded by the Instituto de Salud Carlos III. This work was carried out at the Esther Koplowitz Centre.

The effect of dietary supplementation with $n-3$ polyunsaturated fatty acids on the synthesis of interleukin- 1 and tumor necrosis factor by mononuclear cells. N. Engl. J. Med. 320, 265-271.

Ferrante, A. W. Jr. (2007). Obesityinduced inflammation: a metabolic dialogue in the language of inflammation. J. Intern. Med. 262, 408-414.

González-Périz, A., Horrillo, R., Ferré, N., Gronert, K., Dong, B., MoránSalvador, E., Titos, E., MartínezClemente, M., López-Parra, M. Arroyo, V., and Clària, J. (2009). Obesity-induced insulin resistance and hepatic steatosis are alleviated by omega- 3 fatty acids: a role for resolvins and protectins. FASEB J. 23, 1946-1957.

González-Périz, A., Planagumà, A., Gronert, K., Miquel, R., LópezParra, M., Titos, E., Horrillo, R. Ferré, N., Deulofeu, R., Arroyo, V., Rodés, J., and Clària, J. (2006) Docosahexaenoic acid (DHA) blunts liver injury by conversion to protective lipid mediators: protectin D1 and 17S-hydroxy-DHA. FASEB J. 20, 2537-2539.

Gordon, S., and Taylor, P. R. (2005). Monocyte and macrophage heterogeneity. Nat. Rev. Immunol. 5, 953-964.

Haas-Stapleton, E. J., Lu, Y., Hong, S., Arita, M., Favoreto, S., Nigam, S., Serhan, C. N., and Agabian, N. (2007). Candida albicans modulates host defense by biosynthesizing the pro-resolving mediator resolvin E1. PLoS ONE 2, e1316. doi: 10.1371/journal.pone.0001316

Haworth, O., Cernadas, M., Yang, R., Serhan, C. N., and Levy, B. D. (2008) Resolvin E1 regulates interleukin 23, interferon-gamma and lipoxin A4 to promote the resolution of allergic airway inflammation. Nat. Immunol. 9, 873-879.

Hellmann, J., Tang, Y., Kosuri, M., Bhatnagar, A., and Spite, M. (2011). Resolvin D1 decreases adipose tissue macrophage accumulation and improves insulin sensitivity in obesediabetic mice. FASEB J. 25, 23992407.

Hong, S., Gronert, K., Devchand, P. R., Moussignac, R. L., and Serhan, C. N. (2003). Novel docosatrienes and 17Sresolvins generated from docosahexaenoic acid in murine brain, human blood, and glial cells. Autacoids in anti-inflammation. J. Biol. Chem. 278, 14677-14687.

Horrillo, R., González-Périz, A., Martínez-Clemente, M., LópezParra, M., Ferré, N., Titos, E., MoránSalvador, E., Deulofeu, R., Arroyo, V., and Clària, J. (2010). 5-Lipoxygenase activating protein signals adipose tissue inflammation and lipid dysfunction in experimental obesity. $J$. Immunol. 184, 3978-3987.

Kaaman, M., Rydén, M., Axelsson, T., Nordström, E., Sicard, A., Bouloumié, A., Langin, D., Arner, P., and Dahlman, I. (2006). ALOX5AP expression, but not gene haplotypes, is associated with obesity and insulin resistance. Int. J. Obes. 30, 447-452.

Krishnamoorthy, S., Recchiuti, A., Chiang, N., Yacoubian, S., Lee, C. H., Yang, R., Petasis, N. A., and Serhan, C. N. (2010). Resolvin D1 binds human phagocytes with evidence for proresolving receptors. Proc. Natl. Acad. Sci. U.S.A. 26, 1660-1665.

Mantovani, A., Sica, A., and Locati, M. (2007). New vistas on macrophage differentiation and activation. Eur. J. Immunol. 37, 14-16.

Martínez, F. O., Helming, L., and Gordon, S. (2009). Alternative activation of macrophages: an immunologic 
functional perspective. Annu. Rev. Immunol. 27, 451-483.

Martínez-Clemente, M., Ferré, N., González-Périz, A., López-Parra, M., Horrillo, R., Titos, E., MoránSalvador, E., Miquel, R., Arroyo, V., Funk, C. D., and Clària, J. (2010). 5-Lipoxygenase deficiency reduces hepatic inflammation and tumor necrosis factor alpha-induced hepatocyte damage in hyperlipidemiaprone ApoE-null mice. Hepatology 51, 817-827.

Mehrabian, M., Schulthess, F. T., Nebohacova, M., Castellani, L. W., Zhou, Z., Hartiala, J., Oberholzer, J., Lusis, A. J., Maedler, K., and Allayee, H. (2008). Identification of ALOX5 as a gene regulating adiposity and pancreatic function. Diabetologia 51 , 978-988.

Planagumà, A., Titos, E., López-Parra, M., Gaya, J., Pueyo, G., Arroyo, V., and Clària, J. (2002). Aspirin (ASA) regulates 5-lipoxygenase activity and peroxisome proliferator-activated receptor alpha-mediated CINC-1 release in rat liver cells: novel actions of lipoxin A4 (LXA4) and ASA-triggered 15epi-LXA4. FASEB J. 16, 1937-1939.

Schif-Zuck, S., Gross, N., Assi, S., Rostoker, R., Serhan, C. N., and Ariel, A. (2011). Saturatedefferocytosis generates pro-resolving CD11b low macrophages: modulation by resolvins and glucocorticoids. Eur. J. Immunol. 41, 366-379.

Schwab, J. M., Chiang, N., Arita, M., and Serhan, C. N. (2007). Resolvin E1 and protectin D1 activate inflammationresolution programmes. Nature 447 , 869-874.

Serhan, C. N. (2002). Lipoxins and aspirin-triggered 15-epi-lipoxin biosynthesis: an update and role in anti-inflammation and proresolution. Prostaglandins Other Lipid Mediat. 68-69, 433-455.
Serhan, C. N., Chiang, N., and Van Dyke, T. E. (2008). Resolving inflammation: dual anti-inflammatory and pro-resolution lipid mediators. Nat. Rev. Immunol. 8, 349-361.

Serhan, C. N., Clish, C. B., Brannon, J., Colgan, S. P., Chiang, N., and Gronert, K. (2000). Novel functional sets of lipid-derived mediators with antiinflammatory actions generated from omega-3 fatty acids via cyclooxygenase 2-nonsteroidal antiinflammatory drugs and transcellular processing. J. Exp. Med. 192, 1197-1204.

Serhan, C. N., Gotlinger, K., Hong, S., Lu, Y., Siegelman, J., Baer T., Yang, R., Colgan, S. P. and Petasis, N. A. (2006). Antiinflammatory actions of neuroprotectin D1/protectin D1 and its natural stereoisomers: assignments of dihydroxy-containing docosatrienes. J. Immunol. 176, 1848-1859.

Serhan, C. N., Hamberg, M., and Samuelsson, B. (1984). Lipoxins: novel series of biologically active compounds formed from arachidonic acid in human leukocytes. Proc Natl. Acad. Sci. U.S.A. 81, 5335-5339.

Serhan, C. N., Hong, S., Gronert, K., Colgan, S. P., Devchand, P. R., Mirick, G., and Moussignac, R. L. (2002). Resolvins: a family of bioactive products of omega- 3 fatty acid transformation circuits initiated by aspirin treatment that counter proinflammation signals. J. Exp. Med. 196, 1025-1037.

Spite, M., Norling, L. V., Summers, L., Yang, R., Cooper, D., Petasis, N. A., Flower, R. J., Perretti, M., and Serhan, C. N. (2009). Resolvin D2 is a potent regulator of leukocytes and controls microbial sepsis. Nature 461, 1287-1291.

Sun, Y. P., Oh, S. F., Uddin, J., Yang, R., Gotlinger, K., Campbell, E., Colgan,
S. P., Petasis, N. A., and Serhan, C. N. (2007). Resolvin D1 and its aspirintriggered 17R epimer. Stereochemical assignments, anti-inflammatory properties, and enzymatic inactivation. J. Biol. Chem. 282, 9323-9334.

Tilg, H. (2010). The role of cytokines in non-alcoholic fatty liver disease. Dig. Dis. 28, 179-185.

Titos, E., Clària, J., Bataller, R., BoschMarcé, M., Ginès, P., Jiménez, W. Arroyo, V., Rivera, F., and Rodés, J. (2000). Hepatocyte-derived cysteinyl leukotrienes modulate vascular tone in experimental cirrhosis. Gastroenterology 119, 794-805.

Titos, E., Clària, J., Planagumà, A. López-Parra, M., González-Périz, A. Gaya, J., Miquel, R., Arroyo, V., and Rodés, J. (2005). Inhibition of 5lipoxygenase-activating protein abrogates experimental liver injury: role of Kupffer cells. J. Leukoc. Biol. 78, 871-878.

Titos, E., Clària, J., Planagumà, A., López-Parra, M., Villamor, N., Parrizas, M., Carrió, A., Miquel, R., Jiménez, W., Arroyo, V., Rivera, F., and Rodés, J. (2003). Inhibition of 5-lipoxygenase induces cell growth arrest and apoptosis in rat Kupffer cells: implications for liver fibrosis. FASEB J. 17, 1745-1747.

Titos, E., Rius, B., González-Périz, A. López-Vicario, C., Morán-Salvador, E., Martínez-Clemente, M., Arroyo, V., and Clària, J. (2011). Resolvin D1 and its precursor docosahexaenoic acid promote resolution of adipose tissue inflammation by eliciting macrophage polarization toward an M2-like phenotype. J. Immunol. 15, 5408-5418.

Wallace, J. L., and Vong, L. (2008). NSAID-induced gastrointestinal damage and the design of GI-sparing NSAIDs. Curr. Opin. Investig. Drugs 9, 1151-1156.
Wanninger, J., Bauer, S., Eisinger, K., Weiss, T. S., Walter, R., Hellerbrand, C., Schäffler, A., Higuchi, A., Walsh, K., and Buechler, C. (2012). Adiponectin upregulates hepatocyte CMKLR1 which is reduced in human fatty liver. Mol. Cell. Endocrinol. 349, 248-254.

Wisse, E., Braet, F., Luo, D., De Zanger, R., Jans, D., Crabbé, E., and Vermoesen, A. (1996). Structure and function of sinusoidal lining cells in the liver. Toxicol. Pathol. 24, 100-111.

Conflict of Interest Statement: The authors declare that the research was conducted in the absence of any commercial or financial relationships that could be construed as a potential conflict of interest.

Received: 02 July 2012; accepted: 31 July 2012; published online: 20 August 2012.

Citation: Rius B, López-Vicario C, González-Périz A, Morán-Salvador E, García-Alonso V, Clària $J$ and Titos E (2012) Resolution of inflammation in obesity-induced liver disease. Front. Immun. 3:257. doi: 10.3389/fimmu. 2012.00257

This article was submitted to Frontiers in Inflammation, a specialty of Frontiers in Immunology.

Copyright (c) 2012 Rius, López-Vicario, González-Périz, Morán-Salvador, García-Alonso, Clària and Titos. This is an open-access article distributed under the terms of the Creative Commons Attribution License, which permits use, distribution and reproduction in other forums, provided the original authors and source are credited and subject to any copyright notices concerning any thirdparty graphics etc. 